\title{
PROTEIN OLIGOMERIZATION IS THE BIOCHEMICAL PROCESS HIGHLY UP-REGULATED IN PORCINE OOCYTES BEFORE IN VITRO MATURATION (IVM)
}

Sylwia Borys-Wójcik ${ }^{1}$, Ievgenia Kocherova ${ }^{1}$, Piotr Celichowski ${ }^{2}$, Małgorzata Popis ${ }^{1}$, Michal Jeseta ${ }^{3}$, Dorota Bukowska ${ }^{4}$, Paweł Antosik ${ }^{4}$, Michał Nowicki², Bartosz Kempisty ${ }^{1,2,3}$

\begin{abstract}
A wide variety of mechanisms controlling oligomerization are observed. The dynamic nature of protein oligomerization is important for bioactivity control. The oocyte must undergo a series of changes to become a mature form before it can fully participate in the processes associated with its function as a female gamete. The growth of oocytes in the follicular environment is accompanied by surrounding somatic cumulus (CCs) and granulosa cells (GCs). It has been shown that oocytes tested before and after in vitro maturation (IVM) differ significantly in the transcriptomic and proteomic profiles. The aim of this study was to determine new proteomic markers for the oligomerization of porcine oocyte proteins that are associated with cell maturation competence. The Affymetrix microarray assay was performed to examine the gene expression profile associated with protein oligomerization in oocytes before and after IVM. In total, 12258 different transcriptomes were analyzed, of which 419 genes with lower expression in oocytes after IVM. We found 9 genes: GJA1, VCP, JUP, MIF, MAP3K1, INSR, ANGPTL4, EIF2AK3, DECR1, which were significantly down-regulated in oocytes after IVM (in vitro group) compared to oocytes analyzed before IVM (in vivo group). The higher expression of genes involved in the oligomerization of the protein before IVM indicates that they can be recognized as important markers of biological activation of proteins necessary for the further growth and development of pig embryos.
\end{abstract}

Running title: protein oligomerization during IVM

Keywords: pig, oocytes, microarray assays, in vitro maturation (IVM)

\footnotetext{
${ }^{1}$ Department of Anatomy, Poznan University of Medical Sciences, Poznan, Poland

${ }^{2}$ Department of Histology and Embryology, Poznan University of Medical Sciences, Poznan, Poland

${ }^{3}$ Department of Obstetrics and Gynecology, University Hospital and Masaryk University, Brno, Czech Republic

${ }^{4}$ Veterinary Center, Nicolaus Copernicus University, Torun, Poland

* Correspondence: bkempisty@ump.edu.pl

Full list of author information is available at the end of article
} 


\section{Introduction}

The formation of fully mature oocytes occurs through complex biochemical, molecular and metabolic processes called nuclear and cytoplasmic maturation. Nuclear maturation involves chromatin remodeling and changes in the chromosome configuration, leading to the formation of MII oocytes. Cytoplasmic maturation is the accumulation of mRNA and proteins in the cytoplasm of the cells, which are matrices for further embryo development [1]. It includes events that complete nuclear maturation, fertilization, as well as early embryogenesis.

In vitro maturation of oocytes (IVM) varies significantly between individual mammalian species. The results of the study prove that although porcine embryo has been successfully grown in vitro, the percentage of gametes that have reached the MII stage after $44 \mathrm{~h}$ of culture is still unsatisfactory [2]. In recent years, several modifications of nutrient supplementation have been introduced to increase the number of pig oocytes that reach the MII stage after IVM. Studies show that only oocytes that are sealed by both cumulus cells (CCs) and granulosa cells (GCs) are able to achieve full maturity [3]. It has been unequivocally demonstrated on several species of mammals, including pigs, that only fully mature gametes allow further embryo growth and development in the perimplantation stages. Although the enzymes involved in the metabolism of mammalian oocytes are mostly known, it is still unclear whether and which ones are involved in the regulation of oocyte maturation capacity [4].

The aim of this study was to investigate differences in RNA expression profiles between porcine oocytes before and after IVM using microarrays in relation to genes responsible for oligomerization of proteins. Therefore, in the presented study we used a model in the form of porcine oocytes to determine the transcriptomic profile of these enzymes in relation to the state of oocyte maturity. In our experiment, we focused on target genes such as GJA1, VCP, JUP, MIF, MAP3K1, INSR, ANGPTL4, EIF2AK3, and $D E C R 1$, which expression values differ significantly depending on the stage of oocyte maturation.

\section{Material and Methods Animals}

A total of 45 pubertal crossbred Landrace gilts, with median age of 170 days and weight of $98 \mathrm{~kg}$, were used in this study. The animals were bred under the same conditions.

\section{Collection of porcine ovaries and cumulus- oocyte-complexes (COCs)}

The ovaries and reproductive tracts were recovered at slaughter and transported to the laboratory within $10 \mathrm{~min}$. at $38^{\circ} \mathrm{C}$. in $0.9 \% \mathrm{NaCl}$. To provide optimal conditions for subsequent oocyte matura- tion and fertilization in vitro, the ovaries of each animal were placed in 5\% fetal bovine serum solution (FBS; Sigma-Aldrich Co., St. Louis, MO, USA) in PBS. Thereafter, single large follicles $(>5 \mathrm{~mm})$ were opened, in a sterile Petri dish, by puncturing with a 5-mL syringe and 20-G needle, to recover the COCs. The COCs were washed three times in modified PBS supplemented with $36 \mu \mathrm{g} / \mathrm{mL}$ pyruvate, $50 \mu \mathrm{g} / \mathrm{mL}$ gentamicin, and $0.5 \mathrm{mg} / \mathrm{mL}$ BSA (Sigma-Aldrich, St. Louis, MO, USA). COCs were selected under an inverted microscope- Zeiss, Axiovert 35 (Lübeck, Germany), counted, and morphologically evaluated with special care, using the scale suggested by Pujol et al. and Le Guienne et al..

Grade I: COCs with a homogeneous cytoplasm and a complete cumulus oophorus

Grade II: COCs with an incomplete but compact cumulus oophorus with more than five layers and a homogeneous cytoplasm

Grade III: COCs with a heterogeneous cytoplasm and more than three cumulus cells layers or more than five layers in some parts.

Grade IV: COCs with cumulus oophorus partly or totally absent and cytoplasm strongly heterogeneous."

Only COCs of grade I with homogeneous cytoplasm and uniform and compact cumulus cells were considered for use in the following steps of the experiment, resulting in the use of a total of 300 grade I oocytes that were determined positive in Brilliant Cresyl Blue (BCB) test.

\section{Assessment of oocyte developmental competence by BCB test}

Brilliant Cresyl Blue (BCB) test, which measures activity of glucose-6-phosphate (G6PDH) enzyme, was used for assessment of oocytes' quality and maturity. The G6PDH enzyme converts BCB stain from blue to colorless. In oocytes that completed their growth, activity of the enzyme decreases and the stain cannot be reduced, resulting in blue oocytes $(\mathrm{BCB}+)$. To run the BCB staining test, oocytes were washed two times in modified Dulbecco PBS (DPBS) (Sigma-Aldrich, St. Louis, MO, USA) supplemented with $50 \mathrm{IU} / \mathrm{mL}$ penicillin, $50 \mu \mathrm{g} / \mathrm{mL}$ streptomycin (Sigma-Aldrich, St. Louis, MO, USA), 0.4\% [w/v] BSA, $0.34 \mathrm{mM}$ pyruvate, and $5.5 \mathrm{mM}$ glucose (DPBSm). Thereafter, they were treated with $13 \mu \mathrm{M}$ BCB (Sigma-Aldrich, St. Louis, MO, USA) diluted in DPBSm at $38.5^{\circ} \mathrm{C}, 5 \% \mathrm{CO}_{2}$ in air for $90 \mathrm{~min}$. After treatment, the oocytes were transferred to DPBSm and washed two times. During the washing procedure, the oocytes were examined under an inverted microscope and classified as either having stained blue $\left(\mathrm{BCB}^{+}\right)$or remained colorless $\left(\mathrm{BCB}^{-}\right)$. Immature oocytes have compact cumulus cell layers which need to be removed for further oocyte evaluation, regardless if the oocyte will be later analyzed as "before IVM" group or set to undergo IVM. Therefore, the $\mathrm{BCB}^{+} \mathrm{COCs}$ were first incubated with bo- 
vine testicular hyaluronidase (Sigma-Aldrich, St. Louis, MO, USA) for $2 \mathrm{~min}$ at $38^{\circ} \mathrm{C}$, to separate cumulus and granulosa cells. Afterwards, cells were removed by vortexing the $\mathrm{BCB}^{+}$oocytes in $1 \%$ sodium citrate buffer and by mechanical displacement using a small-diameter glass micropipette. Only the granulosa-cell-free $\mathrm{BCB}^{+}$oocytes were used for subsequent IVM, microarray and RT-qPCR analysis. Overall, 150 immature oocytes were qualified as "before IVM", analyzed straight after the COC shedding, with the rest directed for in vitro maturation ("after IVM") and subsequent analysis.

\section{In vitro maturation of porcine COCs}

After the first BCB test, the blue stained COCs $\left(\mathrm{BCB}^{+}\right)$were cultured in Nunclon ${ }^{\mathrm{TM}} \Delta 4$-well dishes in $500 \mu \mathrm{L}$ standard porcine IVM culture medium, TCM199 (tissue culture medium), with Earle's salts and L-glutamine (Gibco BRL Life Technologies, Grand Island, NY, USA), supplemented with $2.2 \mathrm{mg} / \mathrm{mL}$ sodium bicarbonate (Nacalai Tesque, Inc., Kyoto, Japan), $0.1 \mathrm{mg} / \mathrm{mL}$ sodium pyruvate (Sigma-Aldrich, St. Louis, MO, USA), $10 \mathrm{mg} / \mathrm{mL}$ BSA (bovine serum albumin), (Sigma-Aldrich, St. Louis, MO, USA), 0.1 $\mathrm{mg} / \mathrm{mL}$ cysteine (Sigma-Aldrich, St. Louis, MO, USA), $10 \%(\mathrm{v} / \mathrm{v})$ filtered porcine follicular fluid and gonadotropin supplements at final concentrations of $2.5 \mathrm{IU} / \mathrm{mL}$ hCG (Ayerst Laboratories, Inc., Philadelphia, PA, USA) and 2.5 IU/mL eCG (Intervet, Whitby, ON, Canada). Wells were covered with mineral oil overlay and cultured at $38^{\circ} \mathrm{C}$, under $5 \% \mathrm{CO} 2$, for $22 \mathrm{~h}$, and then for additional $22 \mathrm{~h}$ in medium without hormones. After maturation, the BCB staining test was performed again and $\mathrm{BCB}+$ oocytes were used for further molecular analyses. Based on visual analysis under inverted microscope, around 70\% of oocytes were determined as BCB+. This makes the final number of "after IVM" oocytes around 105 out of the initial 150 .

\section{RNA extraction from porcine oocytes}

Total RNA was extracted from all the samples (both before and after IVM), using TRI Reagent (Sigma, St Louis, MO, USA) and RNeasy MinElute cleanup Kit (Qiagen, Hilden, Germany). The amount of total mRNA was determined from the optical density at $260 \mathrm{~nm}$, while the RNA purity was estimated using the $260 / 280 \mathrm{~nm}$ absorption ratio (higher than 1.8) (NanoDrop spectrophotometer, Thermo Scientific, ALAB, Poland). The RNA integrity and quality were checked on a Bioanalyzer 2100 (Agilent Technologies, Inc., Santa Clara, CA, USA). The resulting RNA integrity numbers (RINs) were between 8.5 and 10 with an average of 9.2 (Agilent Technologies, Inc., Santa Clara, CA, USA). The RNA in each sample was diluted to a concentration of 100 $\mathrm{ng} / \mu \mathrm{L}$ with an OD260/OD280 ratio of 1.8/2.0. From each RNA sample, 100 ng of RNA was taken for the microarray analysis. The remaining amount of isolated RNA was used for RT-qPCR study.

\section{Microarray expression analysis and statistics}

Experiments were performed in three replicates. Total RNA (100 ng) from each pooled sample was subjected to two round sense cDNA amplification (Ambion® WT Expression Kit). The obtained cDNA was used for biotin labeling and fragmentation by Affymetrix GeneChip® WT Terminal Labeling and Hybridization (Affymetrix). Biotin-labeled fragments of cDNA $(5.5 \mu \mathrm{g})$ were hybridized to Affymetrix ${ }^{\circledR}$ Porcine Gene 1.1 ST Array Strip $\left(48^{\circ} \mathrm{C} / 20\right.$ h). Next, the microarrays were washed and stained according to the technical protocol, using Affymetrix GeneAtlas Fluidics Station. The array strips were scanned employing Imaging Station of GeneAtlas System. The preliminary analysis of the scanned chips was performed using Affymetrix GeneAtlasTM Operating Software. The quality of gene expression data was checked according to quality control criteria provided by the software. Obtained CEL files were imported into downstream data analysis software.

All analyzes were performed using BioConductor software, based on the statistical R programming language. For background correction, normalization and summation of raw data, the Robust Multiarray Averaging (RMA) algorithm implemented in "affy" package of BioConductor was applied. Biological annotation was taken from BioConductor "oligo" package where annotated data frame object was merged with normalized data set, leading to a complete gene data table. Statistical significance of analyzed genes was performed by moderated t-statistics from the empirical Bayes method. Obtained p-value was corrected for multiple comparisons using the Benjamini and Hochberg's false discovery rate. The selection of genes with significantly changed expression was based on p-value beneath 0.05 and expression fold higher than $|2|$.

Functional annotation clustering of differentially expressed genes was performed using DAVID (Database for Annotation, Visualization, and Integrated Discovery). Gene symbols for up- or down-regulated genes from each of the compared groups were loaded to DAVID by "RDAVIDWebService" BioConductor package. For further analysis, the enriched GO BP terms which have at least 5 genes and p-value (Benjamini) $<0.05$ were chosen. The enriched GO BP terms were subjected to hierarchical clusterization algorithm and presented as heatmaps.

Interactions between chosen differentially expressed genes/proteins belonging to investigated GO BP terms were investigated by STRING10 software (Search Tool for the Retrieval of Interacting Genes). A list of gene names was used as a query for interaction prediction. Searching criteria based on co-occurrences of genes/proteins in scientific texts (text mining), co-expression and experimentally observed interactions. The results of such analysis generated gene/protein interaction network where 
the intensity of the edges reflects the strength of the interaction score. Besides interaction prediction, STRING also allowed us to perform functional enrichment of GO BP terms based on previously uploaded gene sets.

Finally, the functional interaction between genes that belong to the chosen GO BP terms were investigated by REACTOME FIViz application to the Cytoscape 3.6.0 software. The ReactomeFIViz app is designed to find pathways and network patterns related to cancer and other types of diseases. This app accesses the pathways stored in the Reactome database, allowing to do pathway enrichment analysis for a set of genes, visualize hit pathways using manually laid-out pathway diagrams directly in Cytoscape and investigate functional relationships among genes in hit pathways. The app can also access the Reactome Functional Interaction (FI) network, a highly reliable, manually curated, pathway-based protein functional interaction network covering over $60 \%$ of human proteins.

\section{Ethical approval}

The research related to animal use has been complied with all the relevant national regulations and instructional policies for the care and use of animals. Bioethical Committee approval no. 83/2012/ DNT.

\section{Results}

The whole transcriptome profiling by Affymetrix microarray allowed us to analyze the gene expression changes in freshly isolated oocytes, before in vitro procedure ("before IVM"), in relation to after in vitro maturation ("after IVM"). The expression of 12258 porcine transcripts has been examined by Affymetrix $®$ Porcine Gene 1.1 ST Array. Genes with a fold change higher than $|2|$ and with a corrected p-value lower than 0.05 were considered as differentially expressed. This set of genes consisted of 419 different transcripts. Subsequently, the genes were used for identification of significantly enriched GO BP terms.

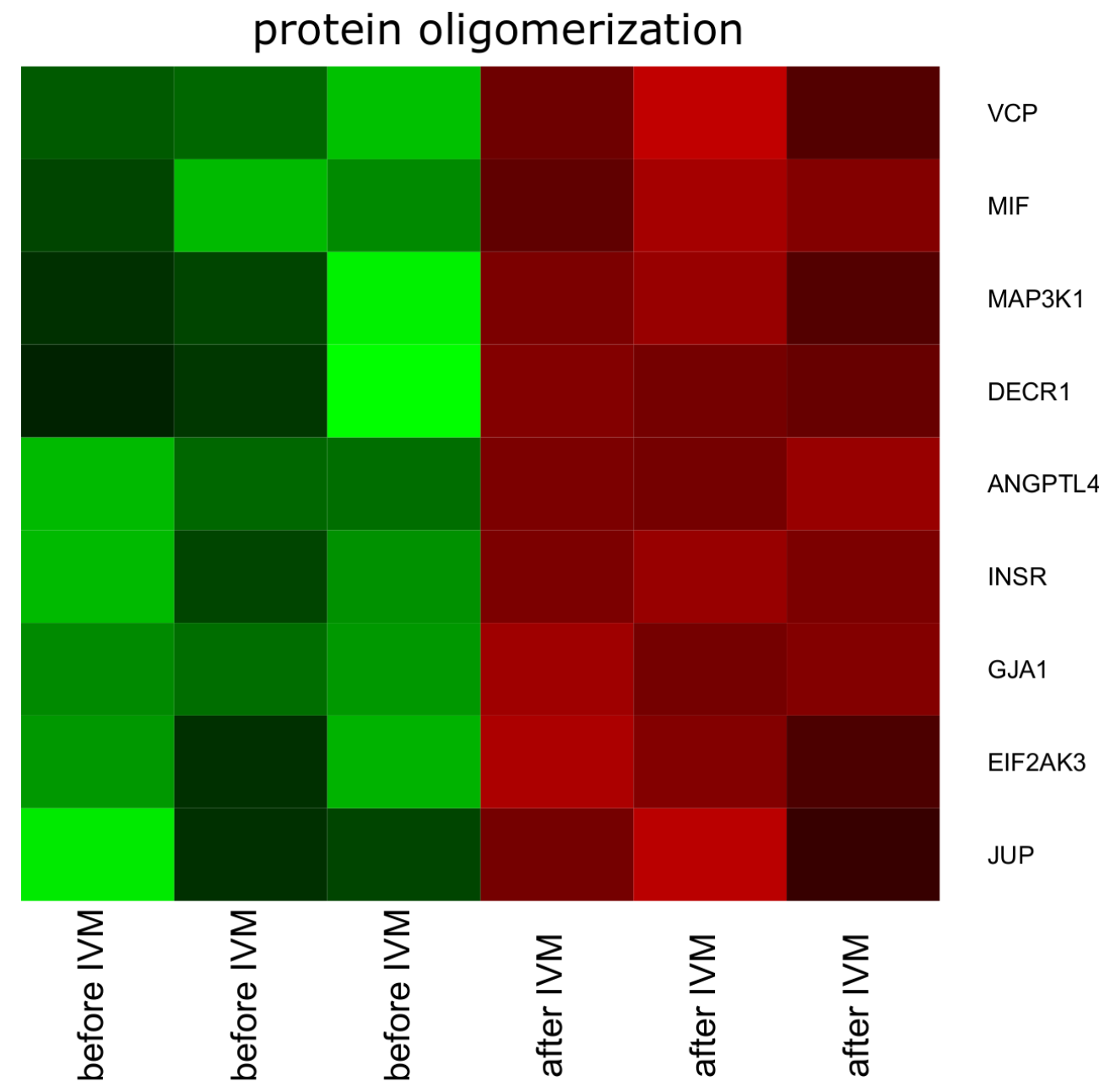

FIGURE 1 Heatmap representations of differentially expressed genes belonging to the "protein oligomerization" GO BP term. Arbitrary signal intensity acquired from microarray analysis is represented by colors (green, higher; red, lower expression). Log2 signal intensity values for any single gene were resized to Row Z-Score scale (from -2, the lowest expression to +2 , the highest expression for a single gene) 
TABLE 1 Gene symbols, fold changes in expression, Entrez gene IDs and corrected p-values of studied genes

\begin{tabular}{c|c|c|c}
\hline Gene Symbol & Fold Change & P Value & Entrez Gene ID \\
\hline GJA1 & 0.206907 & 0.000108 & 100518636 \\
\hline VCP & 0.435612 & 0.007402 & 397524 \\
\hline JUP & 0.441587 & 0.034615 & 397592 \\
\hline MIF & 0.375718 & 0.002882 & 397412 \\
\hline MAP3K1 & 0.368765 & 0.024748 & 396617 \\
\hline INSR & 0.316016 & 0.001913 & 396755 \\
\hline ANGPTL4 & 0.183631 & 0.000513 & 397628 \\
\hline EIF2AK3 & 0.41889 & 0.008422 & 100513348 \\
\hline DECR1 & 0.415159 & 0.042461 & 503544 \\
\hline
\end{tabular}

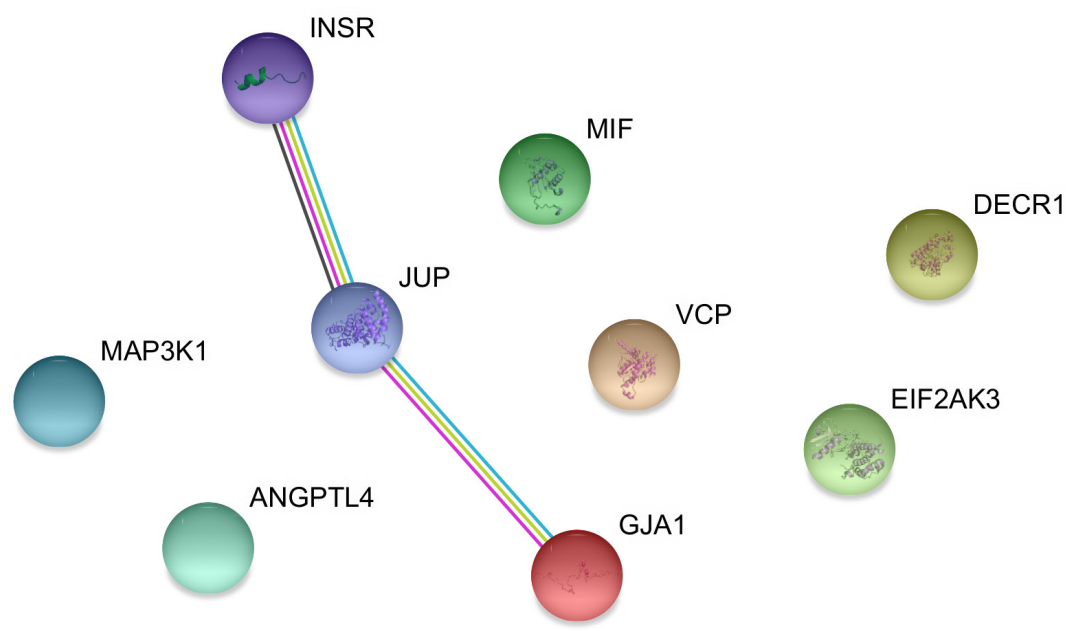

Known interaction

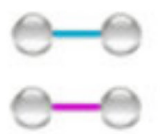

\section{from curated databases experimentally determined}

Predicted interactions

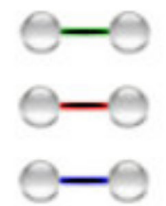

\section{gene neighborhood}

gene fusions

gene co-occurrence
Others

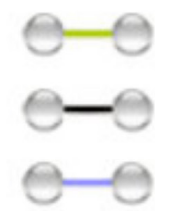

textmining

co-expression

protein homology

FIGURE 2 STRING-generated interaction network between genes that belong to the "protein oligomerization" GO BP term. The intensity of the edges reflects the strength of the interaction score

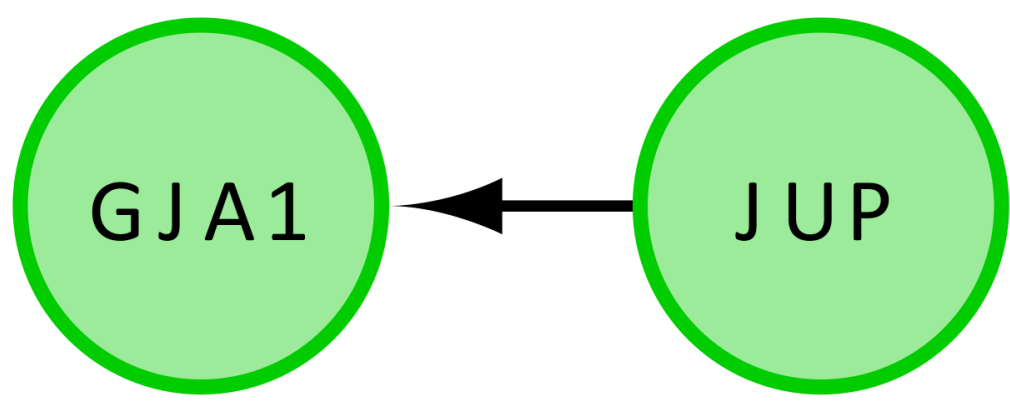

FIGURE 3 Functional interactions (FI) between differentially expressed genes that belong to the "protein oligomerization" GO BP term. In the following figure "->" stands for activating/catalyzing, "-|" for inhibition, “-” for FIs extracted from complexes or inputs and "---" for predicted FIs 
DAVID (Database for Annotation, Visualization, and Integrated Discovery) software was used for extraction of the genes belonging to "protein oligomerization" Gene Ontology Biological Process term (GO BP). We found that 42 genes from this GO BP term were significantly represented in down-regulated gene sets. These sets of genes were subjected to hierarchical clusterization procedure and presented as heatmaps (Fig. 1)

Sets of differentially expressed genes belonging to "protein oligomerization" GO BP term with their official gene symbols, fold changes in expression, Entrez Gene IDs and corrected p-values were shown (Tab. 1).

STRING-generated interaction network was created with differentially expressed genes belonging to the "protein oligomerization" GO BP term. The intensity of the edges reflects the strength of the interaction score (Fig. 2). Finally, the functional interactions between chosen genes were investigated with REACTOME FIViz app to Cytoscape 3.6.0 software. The results were shown in figure 3.

\section{Discussion}

A wide variety of mechanisms controlling oligomerization are observed. The dynamic nature of protein oligomerization is important for bioactivity control. A large part of the cellular proteins is oligomeric. Oligomerization of protein can often be a beneficial feature from the point of view of protein evolution and probably evolved by means of various mechanisms [5]. The main mechanism for the proper maturation and growth of mammalian oocytes is the organization by adequate storage of a large amount of mRNA and proteins. As shown in previous studies of our team [6], both nuclear (reaching MII) and cytoplasmic maturation of oocytes are a key step before successful fertilization, zygote formation, and then the growth and development of early embryos.

Using microarray tests, we analyzed the expression profiles of genes involved in the polymerization of proteins before in vitro maturation (IVM). The data showed substantial up-regulation of several genes in oocytes before IVM, as compared to after IVM. Before IVM, genetic transcripts of genes GJA1, VCP, JUP, MIF, MAP3K1, INSR, ANGPTL4, EIF2AK3, DECR1 were expressed. However, after IVM these genes were down-regulated. This suggests that genes recruited in "protein oligomerization" before porcine oocytes in vitro maturation (IVM), may have a higher level of expression before the oocyte maturation.

A 2,4 -Dienoyl-CoA Reductase 1 (DECR1) gene encodes a mitochondrial enzyme that participates in beta-oxidation and controls the rate of fatty acids metabolism in order to energy production. It participates in the metabolism of unsaturated enoyl-CoA fatty esters having double bonds in both even and odd positions [7]. Congenital deficiency of DECR1 in mammals causes mitochondrial dysfunction, which is manifested by severe encephalopathy with neurological and metabolic disorders [8]. Excessive lipid accumulation in oocytes and the regenerating endometrium reduces fertility by reducing embryonic survival and increasing inflammatory changes [9].

The results of our study have also shown decreased expression of Angiopoietin-Like 4 (ANGPTL4) gene in porcine oocytes after IVM, as compared to those analyzed before IVM. ANGPTL4 encodes a glycosylated secreted protein containing the C-terminal domain of fibrinogen. Peroxisome proliferation activators induce an encoded protein that acts as a hormone in serum. The ANGPTL4 expression product regulates glucose homeostasis, lipid metabolism, and insulin sensitivity. It also acts as apoptosis-inducing factor - it can prevent metastasis by inhibiting vascular growth and invading cancer cells. The reduced expression of the ANGPTL4 gene results in type II diabetes [10]. AlEdani et al. indicated that in human cumulus cells collected from the group of $>37$ years old, genes related to angiogenesis, including ANGPTL4, have been significantly hyper-pressed, compared to the patients from the two younger groups. Angiogenesis plays an important role in the late stages of folliculogenesis by providing nutrients and oxygen to the growing follicles. The presented results indicate that overexpression of ANGPTL4 may be caused by insufficient oxygen (a hypoxic factor) [11].

The macrophage migration inhibitory factor $(M I F)$ is a regulator of innate and acquired immunity. MIF has a significant effect on the response and behavior of macrophages and lymphocytes. The results of many research groups have indicated that the MIF cytokine also plays key roles in reproductive biology [12]. The MIF protein has been identified in a number of reproductive tissues of mammals including an ovary, fallopian tube, uterus and placenta [13]. Many research groups determined the expression of MIF in female reproductive tissues - evidence for expression in follicular fluid and granular cells was obtained in mice and humans. The function performed by MIFs in the ovary and fallopian tube has not yet been clearly defined. However, administration of anti-MIF antibodies to mice significantly reduced the follicles development and maturation. In the case of cattle, it was shown that MIF maintains a population of macrophages in the early yellow body. In humans, pigs and cattle, the presence of MIF in myometrium cells has also been demonstrated [14].

Valosin Containing Protein (VCP) also known as p97 / CDC48 is a member of the AAA ATPase family. VCP maintains the homeostasis of cellular proteins and is responsible for regulating the expression of proteins associated with DNA replication, mitosis, protein degradation, endocytosis, and cell fusion. The results of the conducted studies showed the 
expression of VCP in embryonic mouse gonads and p97 escalation during particular stages of spermatogenesis [15]. Capkova et al. presented evidence that the intra-acrosomal VCP protein is reduced in asthenozoospermics in comparison to healthy men. This result indicates the validity of the use of VCP as a marker for the evaluation of sperm quality in reproductive medicine [16]. Müller et al. demonstrated that the ablation of the VCP gene in mice leads to early mortality of the embryos. This result indicates that p97 coordinates cell proliferation and determines survival, therefore it is necessary for the proper development of mammals [17]. VCP is involved in the regulation of transcription in different biological contexts, suggesting a broad function in gene expression. The decrease in VCP expression after IVM in our study also indicates its involvement in oocyte maturation.

Eukaryotic translation initiation factor 2-alpha kinase $(E I F 2 A 3 K)$, otherwise known as PERK is phosphorylated in response to environmental stress. Translation initiation is stopped, after phosphorylation of eIF2-alpha, which leads to the reduced exchange of GDP to GTP [18]. Harding et al. showed that misfolded proteins remarkably suppress the synthesis of proteins in the endoplasmic reticulum (ER). This process is accompanied by increased phosphorylation of eIF2-alpha in response to extracellular stress [19]. However, they studied the effect of chemical stress in the ER, which leads to translation initiation suppression. Finally, data showed that $E I F 2 A 3 K$ plays an important role in ER adaptation to stress in mammalian cells in vitro [19]. Recently, Lin et al. have focused on the important role of the EIF2A3K kinase signaling pathway in maintaining the proper balance between cell survival and apoptosis [20]. The upregulation of EIF2AK3 in porcine oocytes before IVM can be a substantial indicator of ER stress during gametes in vitro maturation.

Another marker of oocyte maturation status is the expression level of Gap Junction Protein Alpha 1 gene (GJA1). GJA1 belongs to the connexin gene family. The GJA1 protein is an element of the gap junctions, providing an intercellular diffusion path for low molecular weight molecules. The coded protein plays a key role in the synchronization of cardiac contractions and embryonic development through the formation of connections in the heart [21]. Bidirectional intracellular communication between oocytes and cumulus cells is crucial for the proper oocyte maturation [22]. GJA1 is characterized by the ability to activate stopped development of antral follicles, which is why Wang et al. proposed the GJA1 as a new gene marker for oocyte maturation [23]. GJA1 controls the exchange of cAMP and cGMP between cumulus cells and oocytes [24]. According to the results of our research, it can be therefore speculated that GJA1 decreases expression in IVM oocytes as a candidate for oocyte maturity biomarker.
Upregulated expression of Insulin Receptor (INSR) before IVM is correlated with the expression of other genes, which are related to oocyte nuclear maturation process. INSR is located in all ovarian tissues, i.a. in the granulosa and theca cells in bovine species. Insulin is a peptide hormone that plays an important role in the ovary, as a mediator of development of follicles and oocyte maturation [25]. The research results indicate that supplementation of the culture medium with insulin and FSH results in an increased level of INSR mRNA [26]. INSR gene mutations are manifested by disturbed ovulation, as well as oocyte maturation dysfunction [27]. The above information indicates unambiguously that INSR is an important factor in the proper development of oocytes.

Another tested gene was Junction Plakoglobin (JUP). The cytoplasmic protein encoded by this gene is the only known common component of the submembrane desmosomes, as well as their indirect connections. The JUP protein is a member of the catenin family, it is also characterized by the ability to create separate complexes with cadherins and desmosomal cadherins. The reduction of plakoglobin in breast cancer cells increases cell proliferation, while its overexpression limits the proliferative potential of normal cells in vivo or reduces the rate of tumor cells growth in vitro [28]. Mutations in the human JUP gene cause myocardial disease (arrhythmogenic right ventricular cardiomyopathy (ARVC)), which often leads to sudden cardiac death. Inactivation of the JUP gene in mice results in embryo lethality due to a cardiac rupture [29]. In this study, the expression profile of JUP in porcine oocytes before and after IVM has been described, since there is no data indicating its role in mammalian oocyte maturation. Our results indicated higher JUP transcript levels in porcine oocytes before IVM.

The last analyzed gene was Mitogen-Activated Protein Kinases 3 and 1 (MAP3K1). MAP3K1 is involved in the regulation of folliculogenesis and oogenesis. It is also a kinase that stimulates the growth of the cumulus-oocyte complex (COC) and oocyte maturation [30]. This member of intracellular signaling kinases showed decreased mRNA expression after in vitro maturation in our study. Recently $\mathrm{Ou}$ et al. have presented new findings showing the role of MAPK kinase in the maturation of mammalian oocytes. They reported that the role of MAPK is the activation of protein kinase 2 (MK2) in the regulation of porcine oocyte meiotic progression. The authors observed that inhibition or knockdown of the MK2 protein leads to mitotic spindle defects and arrests gametes at GV or prometaphase I/metaphase I stage. Additionally, they suggested that MK2 participates in the spindle organization, as well as in chromosome, microtubule, and kinetochore cellular architecture. Finally, they suggested that MK2 may be recognized as the main factor of porcine oocyte meiotic competence. The current study showed that cell stresses, growth factors, and hor- 
mones may activate $M A P 3 K 1$ and cause downstream signals such as the JNK and ERK $1 / 2$ [31]. The results of recently published studies show that deletion or silencing of MAP3K1 leads to cell cycle arrest or cell death. Silencing the expression of $M A P 3 K 1$ leads to apoptosis of granulosa cells, indicating that MAP3K1 kinase is essential for the survival of GCs [32]. Moreover, MAP3K1 controls the eyelid closure in the early stage of fetal development [33].

As a result of our research, we suggest that all of the presented factors are involved in the in vitro maturation of oocytes.

\section{Corresponding author}

Bartosz Kempisty PhD, Department of Histology and Embryology, Department of Anatomy, Poznań University of Medical Sciences, 6 Święcickiego St., 60-781 Poznań, Poland Tel./Fax: +48 618546418 /+48 61 8546440, e-mail: bkempisty@ump.edu.pl.

\section{Conflict of interest statement}

The authors declare they have no conflict of interest.

\section{References}

1. Watson AJ. Oocyte cytoplasmic maturation: a key mediator of oocyte and embryo developmental competence. J Anim Sci 2007;85:E1-3; DOI:10.2527/jas.2006-432.

2. Agung B, Otoi T, Fuchimoto D, Senbon S, Onishi A, Nagai T. In vitro fertilization and development of porcine oocytes matured in follicular fluid. J Reprod Dev. 2013;59:103-6.

3. Chang CL, Wang H-S, Soong Y-K, Huang SY, Pai SY, Hsu SYT. Regulation of oocyte and cumulus cell interactions by intermedin/adrenomedullin 2. J Biol Chem. 2011;286:43193-203; DOI:10.1074/jbc.M111.297358.

4. Palma GA, Argañaraz ME, Barrera AD, Rodler D, Mutto AÁ, Sinowatz F. Biology and biotechnology of follicle development. ScientificWorldJournal. 2012;2012:938138; DOI:10.1100/2012/938138.

5. Liu S. A review on protein oligomerization process. Int J Precis Eng Manuf. 2015;16:2731-60; DOI:10.1007/s12541-015-0349-x.

6. Celichowski P, Nawrocki MJ, Dyszkiewicz-Konwińska M, Jankowski M Budna J, Bryja A, Kranc W, Borys S, Knap S, Ciesiółka S, Jeseta M, Piasecka-Stryczyńska K, Khozmi R, Bukowska D, Antosik P, Brüssow KP, Bruska M, Nowicki M, Zabel M, Kempisty B. "Positive Regulation of RNA Metabolic Process" Ontology Group Highly Regulated in Porcine Oocytes Matured In Vitro: A Microarray Approach. BioMed Res Int. 2018;2018:2863068; DOI:10.1155/2018/2863068.

7. Marques E, Nkrumah JD, Sherman EL, Moore SS. Polymorphisms in positional candidate genes on BTA14 and BTA26 affect carcass quality in beef cattle. J Anim Sci. 2009;87:2475-84; DOI:10.2527/jas.2008-1456.

8. Davoli R, Fontanesi L, Braglia S, Russo V. A missense mutation in the porcine mitochondrial 2,4-dienoyl CoA reductase 1 (DECR1) gene and linkage mapping of this locus to chromosome 4; Anim Genet. 2002;33:73-5.

9. Wathes DC, Clempson AM, Pollott GE. Associations between lipid metabolism and fertility in the dairy cow. Reprod Fertil Dev. 2012;25:48-61; DOI:10.1071/RD12272.

10. Biterova E, Esmaeeli M, Alanen HI, Saaranen M, Ruddock LW. Structures of Angptl3 and Angptl4, modulators of triglyceride levels and coronary artery disease. Sci Rep. 2018;8:6752; DOI:10.1038/ s41598-018-25237-7.

11. Al-Edani T, Assou S, Ferrières A, Bringer Deutsch S, Gala A, Lecellier C-H, Aït-Ahmed O, Hamamah S. Female aging alters expression of human cumulus cells genes that are essential for oocyte quality. BioMed Res Int. 2014;2014:964614; DOI:10.1155/2014/964614.

12. Groh KJ, Schönenberger R, Eggen RIL, Segner H, Suter MJ-F. Analysis of protein expression in zebrafish during gonad differentiation by targeted proteomics. Gen Comp Endocrinol. 2013;193:210-20; DOI:10.1016/j. ygcen.2013.07.020.

13. Cooke G, Armstrong ME, Donnelly SC. Macrophage migration inhibitory factor (MIF), enzymatic activity and the inflammatory response. BioFactors Oxf Engl. 2009;35:165-8; DOI:10.1002/biof.27.

14. Lopes F, Vannoni A, Sestini S, Casciaro A, Carducci A, Bartolommei S, Toschi P, Ptak G, Cintorino M, Arcuri F. Sheep (Ovis aries) Macrophage Migration Inhibitory Factor: molecular cloning, characterization, tissue distribution and expression in the ewe reproductive tract and in the placenta. Cytokine. 2011;54:315-23; DOI:10.1016/j.cyto.2011.02.013.

15. Franz A, Ackermann L, Hoppe T. Create and preserve: Proteostasis in development and aging is governed by Cdc48/p97/VCP. Biochim Bio- phys Acta BBA - Mol Cell Res. 2014;1843:205-15; DOI:10.1016/j. bbamcr.2013.03.031.

16. Capkova J, Kubatova A, Ded L, Tepla O, Peknicova J. Evaluation of the expression of sperm proteins in normozoospermic and asthenozoospermic men using monoclonal antibodies. Asian J Androl 2016;18:108-13; DOI:10.4103/1008-682X.151400.

17. Müller JMM, Deinhardt K, Rosewell I, Warren G, Shima DT. Targeted deletion of $\mathrm{p} 97$ (VCP/CDC48) in mouse results in early embryonic lethality. Biochem Biophys Res Commun. 2007;354:459-65; DOI:10.1016/j. bbrc.2006.12.206.

18. Shi Y, Vattem KM, Sood R, An J, Liang J, Stramm L, Wek RC. Identification and characterization of pancreatic eukaryotic initiation factor 2 alpha-subunit kinase, PEK, involved in translational control. Mol Cell Biol. 1998;18:7499-509.

19. Harding HP, Zhang Y, Bertolotti A, Zeng H, Ron D. Perk is essential for translational regulation and cell survival during the unfolded protein response. Mol Cell. 2000;5:897-904.

20. Lin JH, Li H, Yasumura D, Cohen HR, Zhang C, Panning B, Shokat KM, Lavail MM, Walter P. IRE1 signaling affects cell fate during the unfolded protein response. Science. 2007;318:944-9; DOI:10.1126/ science.1146361.

21. Basheer WA, Fu Y, Shimura D, Xiao S, Agvanian S, Hernandez DM, Hitzeman TC, Hong T, Shaw RM. Stress response protein GJA1-20k promotes mitochondrial biogenesis, metabolic quiescence, and cardioprotection against ischemia/reperfusion injury. JCI Insight. 2018;3; DOI:10.1172/ jci.insight.121900.

22. Kempisty B, Ziółkowska A, Ciesiółka S, Piotrowska H, Antosik P, Bukowska D, Nowicki M, Brüssow KP, Zabel M. Study on connexin gene and protein expression and cellular distribution in relation to real-time proliferation of porcine granulosa cells. J Biol Regul Homeost Agents. 2014;28:625-35.

23. Wang H-X, Tong D, El-Gehani F, Tekpetey FR, Kidder GM. Connexin expression and gap junctional coupling in human cumulus cells: contribution to embryo quality. J Cell Mol Med. 2009;13:972-84; DOI:10.1111/j.1582-4934.2008.00373.x.

24. Richard S, Baltz JM. Prophase I arrest of mouse oocytes mediated by natriuretic peptide precursor C requires GJA1 (connexin-43) and GJA4 (connexin-37) gap junctions in the antral follicle and cumulus-oocyte complex. Biol Reprod. 2014;90:137; DOI:10.1095/ biolreprod.114.118505.

25. Moller DE, Cohen O, Yamaguchi Y, Assiz R, Grigorescu F, Eberle A, Morrow LA, Moses AC, Flier JS. Prevalence of mutations in the insulin receptor gene in subjects with features of the type A syndrome of insulin resistance. Diabetes. 1994:43:247-55.

26. Chaves RN, Duarte ABG, Rodrigues GQ Celestino JJH, Silva GM, Lopes CAP, Almeida AP, Donato MAM, Peixoto CA, Moura AAA, Lobo CH, Locatelli Y, Mermillod P, Campello CC, Figueiredo JR. The effects of insulin and follicle-simulating hormone (FSH) during in vitro development of ovarian goat preantral follicles and the relative mRNA expression for insulin and FSH receptors and cytochrome P450 aromatase in cultured follicles. Biol Reprod. 2012;87:69; DOI:10.1095/biolreprod.112.099010.

27. Borys S, Brązert M, Jankowski M, Kocherova I, Ożegowska K, Celichowski P, Nawrocki MJ, Kranc W, Bryja A, Kulus M, Jeseta M, Pieńkowski W, Bręborowicz A, Bukowska D, Antosik P, Pawelczyk L, Skowroński MT, Brüssow KP, Bruska M, Zabel M, Nowicki M, Kempisty B. Enzyme linked receptor protein signaling pathway is one of the ontology groups that are highly up-regulated in porcine oocytes before in vitro maturation. J Biol Regul Homeost Agents. 2018;32:1089-103.

28. Procházková J, Kabátková M, Šmerdová L, Pacherník J, Sykorová D, Kohoutek J, Šimečková P, Hrubá E, Kozubík A, Machala M, Vondráček J. Aryl hydrocarbon receptor negatively regulates expression of the plakoglobin gene (jup). Toxicol Sci Off J Soc Toxicol. 2013;134:258-70; DOI:10.1093/toxsci/kft110.

29. Swope D, Li J, Muller EJ, Radice GL. Analysis of a Jup hypomorphic allele reveals a critical threshold for postnatal viability. Genes N Y N 2000. 2012;50:717-27; DOI:10.1002/dvg.22034

30. Fan H-Y, Liu Z, Shimada M, Sterneck E, Johnson PF, Hedrick SM, Richards JS. MAPK3/1 (ERK1/2) in ovarian granulosa cells are essential for female fertility. Science. 2009;324:938-41; DOI:10.1126/science.1171396.

31. Ou X-H, Li S, Xu B-Z, Chen L-N, Jiang M-X, Chen S-Q, Chen N-Q. Mitogen-activated protein kinase-activated protein kinase 2 is a critical regulator of pig oocyte meiotic maturation. Reprod Fertil Dev. 2017;29:22333; DOI:10.1071/RD15150.

32. Cao R, Wu W, Zhou X, Liu K, Li B, Huang X, Zhang Y, Liu H. Let-7g induces granulosa cell apoptosis by targeting MAP3K1 in the porcine ovary. Int J Biochem Cell Biol. 2015;68:148-57; DOI:10.1016/j.biocel.2015.08.011.

33. Xia Y, Karin M. The control of cell motility and epithelial morphogenesis by Jun kinases. Trends Cell Biol. 2004;14:94-101; DOI:10.1016/j. tcb.2003.12.005. 